\title{
Determination of $\mathrm{Y}$-linolenic in Donkey Meat by Near Infrared Spectroscopy and Chemometrics
}

\author{
Xiaoying $\mathrm{Niu}^{1, \mathrm{a}^{*}}$ and Limin Shao ${ }^{2, \mathrm{~b}}$ \\ ${ }^{1}$ College of Quality and Technical Supervision, Hebei University, Baoding 071002, China \\ ${ }^{2}$ College of Mechanical and Electrical Engineering, Hebei Agricultural University, Baoding 071001, \\ China \\ axiaoyingniu@126.com, bshaolm@126.com
}

Keywords: $\gamma$-linolenic; Donkey meat; NIRS; iPLS

\begin{abstract}
Gamma$-linolenic is one kind of polyunsaturated fatty acid (PUFA) essential for human body. The aim of this study was to investigate the feasibility of Near Infrared Spectroscopy (NIRS) to determine the content of $\gamma$-linolenic in donkey meat. The spectra of forty donkey meat samples on the spectral range of 4000-12500 $\mathrm{cm}^{-1}$ were used to develop calibration models with the algorithms of forward iPLS (FiPLS) and backward iPLS (BiPLS). The best calibration is obtained using FiPLS with 80 intervals of the minced sample spectra, the determination coefficient, standard error, and the ratio of performance deviation of cross-validation of which is $0.604,157.3 \mathrm{mg} / 100 \mathrm{~g}$, and 1.60 . The result shows that NIRS combined with chemometrics can predict $\gamma$-linolenic in donkey meat. Further studies are needed to improve the prediction performance of calibration models.
\end{abstract}

\section{Introduction}

Donkey meat is popularly consumed in China due to its high nutritional value and unique flavor. $\Gamma$-linolenic is one kind of polyunsaturated fatty acid (PUFA) essential for human body. The body must get $\gamma$-linolenic from diet because it cannot produce $\gamma$-linolenic on its own. The contribution of $\gamma$-linolenic intake to the diet comes from meat. Donkey meat contains a variety of polyunsaturated fatty acid including linolenic [1]. The quality of donkey meat processing can be improved through the determination of $\gamma$-linolenic. Conventional methods for reliably determining fatty acid (FA) contents in meat, such as gas chromatography (GC), are high time-consuming, laborious, destructive to samples and often requiring use of hazard solvents, which make it impossible for real-time and on-line measurements [2-3]. Near infrared spectroscopy (NIRS) is a modern analysis technique, which can perform quantitative analysis of chemical compositions efficiently, rapidly and environmental friendly. The overtones and combinations of the vibrations of $\mathrm{C}-\mathrm{H}, \mathrm{O}-\mathrm{H}, \mathrm{N}-\mathrm{H}$, and $\mathrm{C}=\mathrm{O}$ as well as some other bonds can be observed in the NIRS range, making it possible for measurements of various parameters in food analysis [4]. In recent years, NIRS was used to determine individual PUFA in pork, beef, mutton, rabbit meat, and chicken and so on [5-15]. But there are no study using NIRS to determine individual PUFA in donkey meat reported as yet. The aim of this study was to use NIRS with chemometrics to predict the content of $\gamma$-linolenic in intact and minced donkey meat samples.

\section{Materials and Methods}

Samples. Fresh donkey meats were purchased from a local butcher's shop in Baoding city on the day of the experiment. At first, skin, excess fat, bruises and other blemishes were removed from several chunks of donkey meat. After trimmed donkey meats were cut into cubes measuring $5 \times 5 \times 2 \mathrm{~cm}$.

A total of forty donkey meat samples were acquired. And after spectral collection of intact sample, samples were minced by an electric meat mincing machine, and then put into a quartz sample cup and compacted to measure spectra of minced samples.

NIRS and Chemical Analysis. NIR diffuse reflectance spectra were collected on the day of sample preparation by a multi-purpose analyzer (MPA) spectrometer (Bruker Optics, Ettlingen, Germany) with 
a $\mathrm{PbS}$ detector and an internal gold background as the reference. The software of OPUS 6.5 (Bruker Optics, Ettlingen, Germany) was used to acquire spectra over a band range of $4000-12500 \mathrm{~cm}^{-1}$ with the resolution of $2 \mathrm{~cm}^{-1}$. Three spectra per sample were measured and the mean spectrum was used to develop calibration models. $\Gamma$-linolenic in donkey meat samples was determined by a gas chromatography with a flame ionisation detection (FID) detector (Agilent 7890A GC, USA). All the experiment was conducted at room temperature.

Chemometrics. Interval Partial Least Squares (iPLS) was used to develop calibration models for quantitative analysis of $\gamma$-linolenic in donkey meat samples. Interval partial least-squares (iPLS) was proposed by Nørgaard et al. for range selection in 2000, which was a graphically oriented approach for local regression modelling of spectral data [16]. Some expansion algorithms of iPLS, such as forward iPLS (FiPLS) and backward iPLS (BiPLS) were also developed and performed successfully [17-18]. In this study, FiPLS and BiPLS was used to select optimal spectral bands for modeling, and all steps were carried out in MATLAB 7.5 (Mathworks, Natick, USA) with a free PLS Toolbox 5.8 (Eigenvector Research, Inc., USA). The predictive ability of models was evaluated by calculating the determination coefficients in calibration and cross-validation (R2), standard error of calibration and cross-validation (SEC, SECV), and the ratio of performance deviation (RPD), i.e. the ratio of the standard deviation of the response variable (SD) to the SECV[19]. The model with highest RPD and lowest SECV would be the best model.

\section{Results and Discussion}

The number of means, standard deviation and range of $\gamma$-linolenic measured by reference method is $179.4 \mathrm{mg} / 100 \mathrm{~g}, 252.1 \mathrm{mg} / 100 \mathrm{~g}$, and $1.1-717.8 \mathrm{mg} / 100 \mathrm{~g}$. The content of $\gamma$-linolenic in samples changes obviously. There are two orders of magnitude between the minimum and maximum. It might be due to the big difference of fat content in samples.

Table 1 calibration and cross validation parameters for quantitative analysis of $\gamma$-linolenic in donkey meat intact samples

\begin{tabular}{cccccccc}
\hline \multirow{2}{*}{ Method } & $\begin{array}{c}\text { No. of } \\
\text { interval }\end{array}$ & \multicolumn{2}{c}{ Calibration } & \multicolumn{3}{c}{ Cross Validation } & \multirow{2}{*}{ Factors } \\
\cline { 2 - 6 } & $\mathbf{S}$ & $\boldsymbol{R}^{\mathbf{2}}$ & $\begin{array}{c}\text { SEC } \\
(\mathbf{m g} / \mathbf{1 0 0 g})\end{array}$ & $\boldsymbol{R}^{\mathbf{2}}$ & $\begin{array}{c}\text { SECV } \\
(\mathbf{m g} / \mathbf{1 0 0 g})\end{array}$ & $\mathbf{R P D c v}$ & \\
\hline \multirow{6}{*}{ FiPLS } & 5 & 0.927 & 67.3 & 0.215 & 240.8 & 1.05 & 10 \\
& 10 & 1.000 & 1.5 & 0.165 & 232.2 & 1.09 & 9 \\
& 20 & 0.429 & 188.1 & 0.199 & 228.1 & 1.11 & 3 \\
& 40 & 0.358 & 199.5 & 0.210 & 223.8 & 1.13 & 2 \\
& $\mathbf{8 0}$ & $\mathbf{0 . 9 9 9}$ & $\mathbf{6 . 3}$ & $\mathbf{0 . 4 9 2}$ & $\mathbf{1 7 8 . 0}$ & $\mathbf{1 . 4 2}$ & $\mathbf{1 0}$ \\
\hline \multirow{6}{*}{ BiPLS } & 5 & - & - & - & - & - & - \\
& 10 & 0.980 & 35.1 & 0.148 & 239.0 & 1.05 & 8 \\
& 20 & 0.984 & 31.4 & 0.337 & 202.7 & 1.24 & 8 \\
& 40 & 0.968 & 44.7 & 0.433 & 187.8 & 1.34 & 8 \\
\hline
\end{tabular}

FiPLS and BiPLS calibration models were developed for $\gamma$-linolenic with intact and minced sample spectra respectively. The performance of models with intervals numbers of 5, 10, 20, 40, and 80 were compared with each other. Then the better calibration models as well as sample types, method including chemometrics and interval numbers used, and the statistics obtained for each calibration are presented in Table 1 and Table 2. The spectra has no correlation with reference data in the BiPLS model with 5 intervals, so the results are not shown in Table 1. With the increase of number of intervals, the performance of iPLS models becomes better. It could be that the smaller intervals contain more 
characteristic of samples. But due to the decrease numbers of variable, some overfittings are shown in the results. The improvements lie in the increase of $\mathrm{R}^{2}$ and RPD, as well as the decrease of SEC and SECV. For intact samples, the performance of 80 intervals (used 1541-1650, 1871-1980, 2311-2420 and 3961-4070) with FiPLS is better than other FiPLS and BiPLS model, which get 0.492 and 178.0 $\mathrm{mg} / 100 \mathrm{~g}$ for $\mathrm{R}^{2}$ of cross-validation and SECV. And for minced samples, also the performance of 80 intervals (used 4621-4730, 4841-4950 and 6381-6490) with FiPLS is better than other FiPLS and BiPLS model, but the $\mathrm{R}^{2}$ of cross-validation and SECV of which model is improved compared with the better one for intact samples. The best calibration for quantitative analysis of $\gamma$-linolenic in donkey meat samples is attained using FiPLS with 80 intervals of the minced sample spectra, the RPDcv value of which is the highest. According to Su et al. [20], the RPDcv value of the best model is 1.6, higher than 1.5 and lower than 2, which indicate that the model can be used to predict $\gamma$-linolenic in donkey meat samples, but the result is not a good calibration, and the prediction performance of the model need to be improved in the further studies.

Table 2 calibration and cross validation parameters for quantitative analysis of $\gamma$-linolenic in donkey meat minced samples

\begin{tabular}{cccccccc}
\hline Method & $\begin{array}{c}\text { No. of } \\
\text { interval }\end{array}$ & \multicolumn{2}{c}{ Calibration } & \multicolumn{3}{c}{ Cross validation } & \multirow{2}{*}{ Factors } \\
\cline { 2 - 6 } & $\mathbf{s}$ & $\boldsymbol{R}^{2}$ & $\begin{array}{c}\text { SEC } \\
(\mathbf{m g} / \mathbf{1 0 0 g})\end{array}$ & $\boldsymbol{R}^{2}$ & $\begin{array}{c}\text { SECV } \\
(\mathbf{m g} / \mathbf{1 0 0 g})\end{array}$ & RPDcv & \\
\hline FiPLS & 5 & 0.955 & 52.6 & 0.273 & 215.6 & 1.17 & 5 \\
& 10 & 1.000 & 4.2 & 0.297 & 210.4 & 1.20 & 7 \\
& 20 & 0.995 & 18.4 & 0.344 & 202.2 & 1.25 & 5 \\
& 40 & 0.993 & 21.2 & 0.521 & 172.4 & 1.46 & 5 \\
& $\mathbf{8 0}$ & $\mathbf{0 . 9 9 1}$ & $\mathbf{2 3 . 1}$ & $\mathbf{0 . 6 0 4}$ & $\mathbf{1 5 7 . 3}$ & $\mathbf{1 . 6 0}$ & $\mathbf{6}$ \\
\hline BiPLS & 5 & 0.762 & 121.5 & 0.271 & 222.4 & 1.13 & 5 \\
& 10 & 0.751 & 124.3 & 0.248 & 225.6 & 1.12 & 5 \\
& 20 & 0.728 & 129.7 & 0.270 & 222.0 & 1.14 & 5 \\
& 40 & 0.834 & 101.4 & 0.354 & 206.9 & 1.22 & 5 \\
& 80 & 0.818 & 106.2 & 0.386 & 201.8 & 1.25 & 5 \\
\hline
\end{tabular}

\section{Conclusion}

The $\gamma$-linolenic in meat is essential for human health. The feasibility of Near Infrared Spectroscopy (NIRS) to determine the content of $\gamma$-linolenic in donkey meat is investigated in this study. The spectra of forty donkey meat samples on the spectral range of $4000-12500 \mathrm{~cm}^{-1}$ were collected, and the reference data were gained by a gas chromatography with flame ionisation detection (FID) detector. The calibration models are developed to predict $\gamma$-linolenic in donkey meat by NIRS and iPLS. The algorithms of forward iPLS (FiPLS) and backward iPLS (BiPLS) with different numbers of interval are compared with each other. And the best model is obtained by the FiPLS with 80 intervals (used 4621-4730, 4841-4950 and 6381-6490) of the minced sample spectra. The determination coefficient of calibration and cross-validation, standard error of calibration and cross-validation, and the ratio of performance deviation of cross-validation of the best model are $0.991,23.1 \mathrm{mg} / 100 \mathrm{~g}, 0.604,157.3$ $\mathrm{mg} / 100 \mathrm{~g}$, and 1.60. The result shows that NIRS combined with chemometrics would be a useful tool to predict $\gamma$-linolenic in donkey meat. Further studies are needed to improve the prediction performance of calibration models. And in order to enhance the adaptability of the model, the sample size should be added too.

\section{Acknowledgements}

This study was supported by the Natural Science Foundation of Hebei Province (C2016201092). 


\section{References}

[1] J. You, Y.K. Luo and Y.C. Zhang: Food Science and Technology, Vol. 34 (2009) No.2, p.118. (In Chinese)

[2] F.F. Tao and Michael Ngadi: Current Opinion in Food Science, Vol. 17 (2017), p.100.

[3] M. Lucarini, A. Durazzo, J. Sánchez del Pulgar, P. Gabrielli and G. Lombardi-Boccia: https://doi.org/10.1016/j.foodchem.2017.11.042.

[4] J.H. Qu, D. Liu, J.H. Cheng, D.W. Sun, J. Ma, H. Pu and X.A. Zeng: Critical Reviews in Food Science and Nutrition, Vol. 55 (2015), p.1939.

[5] L.L. Tao, X.J. Yang, J.M. Deng and X. Zhang: Spectroscopy and Spectral Analysis, Vol. 33 (2013) No.11, p.3002. (In Chinese)

[6] D. Pérez-Marín, E. De Pedro Sanz, J.E. Guerrero-Ginel and A. Garrido-Varo: Meat Science, Vol. 83 (2009) No.4, p.627.

[7] X. Luo, B.P. Liu, X.L. Zhang, G.S. Li and H.J. Qin: Chinese Journal of Analysis Laboratory, Vol. 26 (2007) No.10, p.25. (In Chinese)

[8] A Ripoche and A.S Guillard: Meat Science, Vol. 58 (2001) No.3, p.299.

[9] E. Zamora-Rojas, A. Garrido-Varo, E. De Pedro-Sanz, J.E. Guerrero-Ginel and D. Pérez-Marín: Meat Science, Vol. 95 (2013) No.3, p.503.

[10] N. Prieto, B. Uttaro, C. Mapiye, T.D. Turner, M.E.R. Dugan, V. Zamora, M. Young, E. Beltranena: Meat Science, Vol. 98 (2014) No.4, p.585.

[11]R. Riovanto, M. De Marchi, M. Cassandro and M. Penasa: Food Chemistry, Vol. 134 (2012) No.4, p. 2459.

[12] N. Prieto, M.E.R. Dugan, O. López-Campos, T.A. McAllister, J.L. Aalhus and B. Uttaro: Meat Science, Vol. 90 (2012) No.1, p.43.

[13] N. Prieto, Ó. López-Campos, J.L. Aalhus, M.E.R. Dugan, M. Juárez and B. Uttaro: Meat Science, Vol. 98 (2014) No.2, p. 279.

[14] M. Pla, P. Hernández, B. Ariño, J.A. Ramírez and Isabel Díaz: Food Chemistry, Vol. 100 (2007) No.1, p.165.

[15] R.R. Pullanagari, I.J. Yule and M. Agnew: Meat Science, Vol. 100 (2015), p.156.

[16] Saudland, A., Wagner, J., Nielsen, J. P., Munck, L., Nørgaard, L. and Engelsen, S. B: Applied Spectroscopy, Vol. 54 (2000) No.3, p.413.

[17] Balabin, R. M. and Smirnov, S. V: Analytica Chimica Acta, Vol. 692 (2011) No.1-2, p.63.

[18] Leardi, R. and Nørgaard, L: Journal of Chemometrics, Vol. 18 (2004) No.11, p.486.

[19] A. Teixeiraa, A. Oliveira, K. Paulos, A. Leite, A. Marcia, A. Amorim, E. Pereira, S. Silva and S. Rodrigues: Small Ruminant Research, Vol. 126 (2015), p.40.

[20] H.W. Su, S. Kun, L. Zhang, Q. Zhang, Y.L. Xu, R. Zhang, H.P. Li and B.Z. Sun: Meat Science, Vol. 98 (2014), p.110. 